\title{
CONTINUOUS FLOW SEPARATION OF SUSPENDED CELLS IN HUMAN WHOLE BLOOD USING DIELECTROPHORESIS
}

\author{
Youngdo Jung ${ }^{1}$, Ki-Ho Han ${ }^{2}$, Yoonsu Choi ${ }^{1}$, and A. Bruno Frazier ${ }^{1}$ \\ ${ }^{1}$ Georgia Institute of Technology, Atlanta, Georgia, USA \\ ${ }^{2}$ Inje University, Gimhae, Gyongnam, South Korea
}

\begin{abstract}
This paper is focused on the development of continuous flow dielectrophoretic (DEP) microsystems. The design and fabrication of the continuous flow DEP microsystems are presented along with the experimental characterization of the separation systems using human whole blood as sample material. The significance of this work is the development of the first continuous flow-based DEP microsystems for high-throughput separation of suspended cells in blood by cell type. Experimental results show that an angled-electrode type DEP microsystem can switch the flow stream of blood cells with $96.16 \pm 2.48 \%$ efficiency. Divergent type DEP microseparator can continuously separate out $87.0 \%$ of the red blood cells (RBCs) and 92.1\% of the white blood cells (WBCs), while the convergent type DEP microseparator can separate 93.6\% of the RBCs and $76.9 \%$ of the WBCs suspended in dilute human whole blood with a high conductivity of $17 \mathrm{mS} / \mathrm{cm}$.
\end{abstract}

\section{INTRODUCTION}

Dielectrophoresis uses the electrical polarization of cells in a non-uniform electric field. Depending on the frequency of the applied AC electric field, a cell may feel an attractive force (positive DEP) in the electric field gradient or a repulsive force (negative DEP) in the opposite direction of the field gradient. Most cells experience a positive DEP force in the high frequency range and a negative DEP force in the low frequency region. At the crossover frequency, no DEP forces are exercised on the cells. The crossover frequency is dependent on various cell characteristics, such as the membrane characteristics, internal structures, or the size of cells, and the variability in the crossover frequency of cells is selective enough for DEP microsystems to differentiate between similar cell types. Microfluidic systems using DEP force have been shown to manipulate biological cells successfully using this intrinsic dielectric affinity differentiation and have demonstrated highly selective isolation of target rare cells from the mixture of various blood cells without any tagging [1-4].

However, most of conventional DEP microsystems require several discontinuous steps of retaining, washing and releasing samples. Recently, several continuous DEP microseparators were demonstrated for yeast cells, showing good positive and negative DEP action for viable and nonviable cells $[5,6]$.

The presented continuous flow-based DEP microsystems allow the high-throughput separation of suspended cells in human whole blood by cell type and would aid in the development of blood sample preparation and analysis systems for cell research and medical applications.

\section{METHOD}

\section{Design Method}

The conceptual view of an angled-electrode type DEP microsystem is shown in Fig. 1 (a) along with cell streams during operation. The input sample fluid is focused by two sided sheath flow (buffer \#1, \#2) and down stream comb-type angled electrodes. The electrodes are centered on the input sample channel and the outlet \#1 channel with decreasing comb tooth length from the input to the output of the microseparator along the length of DEP chamber.
Without any force acting on cells, all cells travel with laminar flow and are collected into the outlet \#2 (blue-line). Under a high gradient electric field created by the alternating electrodes, the cells experiencing positive DEP force are attracted toward the electrode edges, thus the cell stream is deflected toward outlet \#1 (red-line), while the cells experiencing negative DEP force are levitated above the surface and swept with laminar flow to outlet \#2 (blue-line). To manipulate biological cells more efficiently in a switching operation, the DEP system utilizes an induced hopping movement of the blood cells between neighboring electrodes by adjusting the release phase and attraction phase properly. Individual cell movement during one hopping period is described in Fig.1 (b).
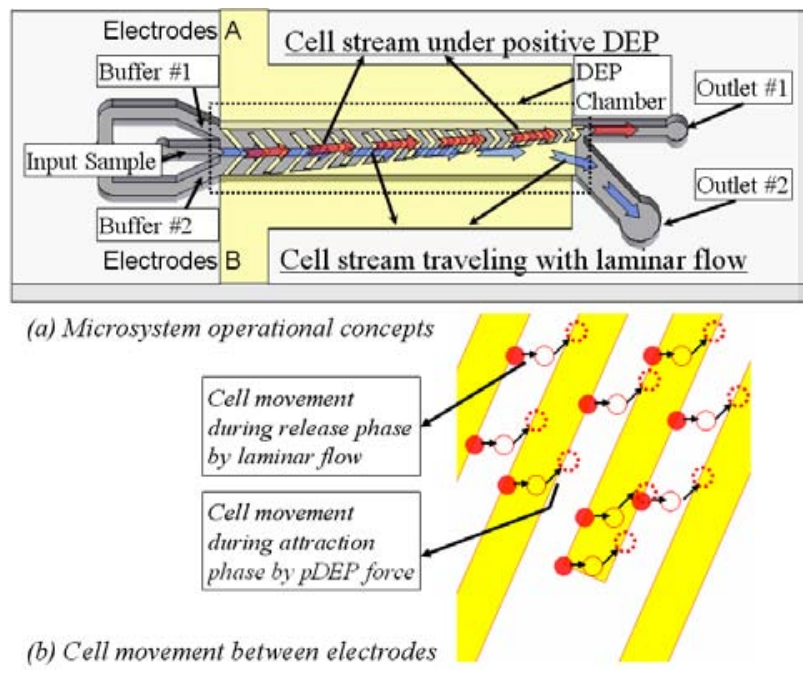

Figure 1: Operation principle of an angled-electrode type continuous DEP microsystem

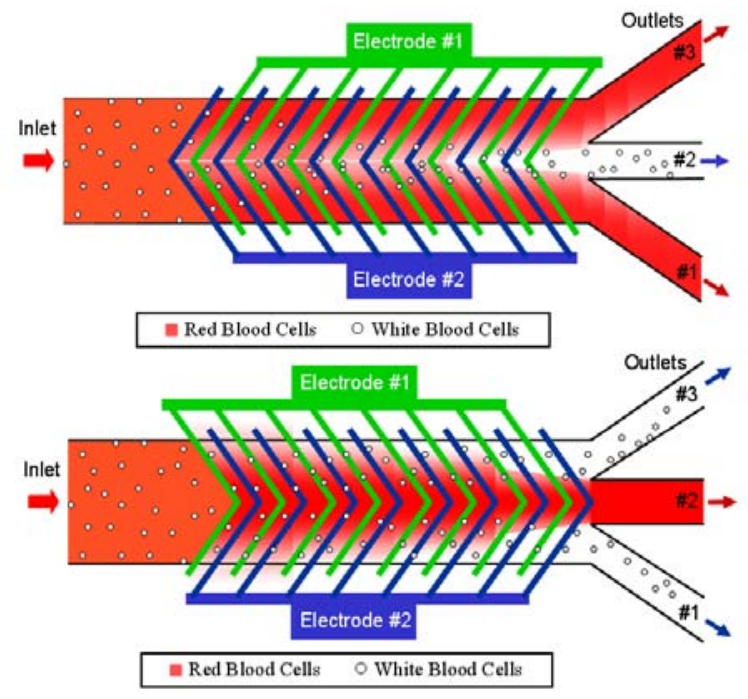

Figure 2: Operation principle of divergent and convergent type continuous DEP microsystems 
The design of the divergent and convergent type DEP microseparators is shown in Fig. 2. At the crossover frequency of WBCs which is usually lower than that of RBCs, the DEP force on WBCs is much weaker than the DEP force on RBCs. Thus, weakly forced WBCs are diffused away from highly dense stream of RBCs. The RBCs and WBCs are continuously separated as the whole blood passes through the microchannel of the DEP microseparators.

\section{Fabrication Method}

The angled-electrode type DEP microsystem consisted of a glass microchannel, planar electrodes and microfluidic interfaces / system packaging. $\mathrm{Cr} / \mathrm{Au}$ masking layers were sputtered on both sides of glass substrates (Schott Borofloat glass) with a DC sputterer. A $50 \mu \mathrm{m}$ deep microchannel was etched through standard photolithography processes followed by HF wet etching of the glass for 30 minutes (Fig. 3 (a)). After removing all $\mathrm{Cr} / \mathrm{Au}$ masking layers for the microchannel etching, additional $\mathrm{Cr} / \mathrm{Au}$ layers for electrodes were sputtered on the surface of the etched glass substrate. The down stream comb-type angled electrodes were defined by another photolithography step using thick negative photoresist (Fig. 3 (b)).

A drilling guide, used to create the inlet and outlet ports in the top glass, the microfluidic interface, and microsystem packaging were designed and fabricated using stereolithography (Fig. 3 (e)).

The inlet and outlet holes along with electrode connection holes were drilled by diamond drill bits using a drilling guide for correct positioning on the top glass substrate. The drilled top glass was aligned and bonded with the patterned bottom glass by introducing UV cure adhesive resin between the two glass substrates by capillary force and curing them under a UV lamp for 30 minutes (Fig. 3 (c)). The microfluidic interface was bonded to the microseparator by repeating the UV adhesive bonding process (Fig. 3 (d)). Finally, two electric wires were connected to the electrode pads through the electrode connection holes using conductive silver paste.

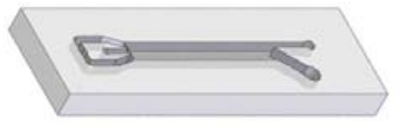

(a) 50 um Channel Etching

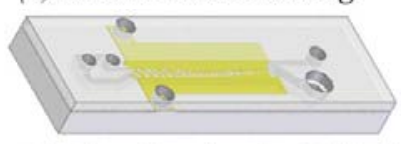

(c) Glass Bonding by UVAdhesive
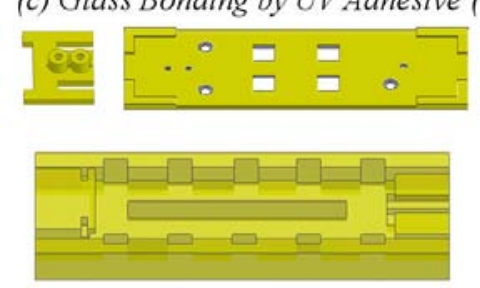

(e) Interconnect, Drilling Guide, and Package

Figure 3: Fabrication process for an angled-electrode type microseparator

The divergent and convergent type DEP microseparators consisted of a PDMS microchannel from SU-8 mold structure, planar electrodes on the glass substrate, and the system packaging. Fig. 4 illustrates the fabrication process for the divergent and convergent type DEP microseparators.

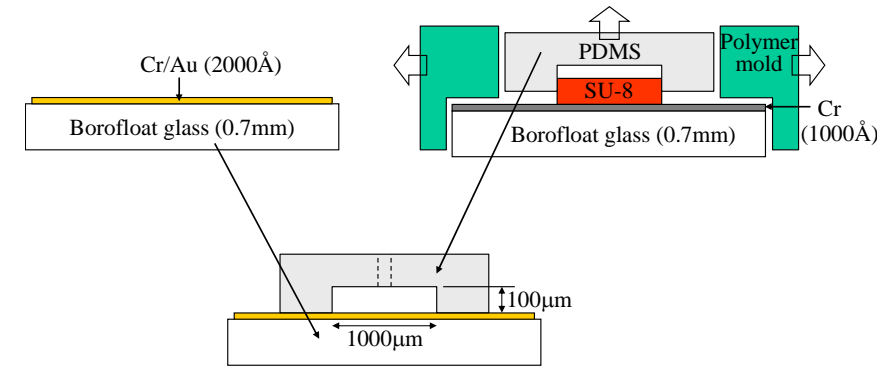

Figure 4: Fabrication process based on glass and PDMS for the divergent and convergent type DEP microseparators

\section{EXPERIMENTAL SETUP}

Human venous blood was collected at on-campus health center under the Institutional Review Board-approved protocol for blood collection for research purposes. The samples were drawn into evacuated glass tubes, containing EDTA as an anticoagulant. 8.5\% $(\mathrm{w} / \mathrm{v})$ sucrose plus $0.3 \%(\mathrm{w} / \mathrm{v})$ dextrose buffer solution was used to dilute human whole blood sample to the ratio of 10:1 and lower the conductance of the media. SYTO-13 fluorescent dye was used to identify the nucleated cells during experiments. The diluted blood sample with SYTO-13 fluorescent dye was incubated at $37^{\circ} \mathrm{C}$ for one hour.

The microseparator was treated with Pluronic ${ }^{\circledR}$ F108 block copolymer surfactant solution to reduce the adhesion of blood cells on the channel surface and washed several times with the buffer solution. The microsystem was assembled into the packaging and the attached electric wires were connected to the function generator output. A $500 \mathrm{uL}$ gas tight glass syringe and a $3 \mathrm{~mL}$ luer lock syringe were used to load the incubated blood sample and the buffer solution, and were connected to capillary tubing. The other ends of the tubing extending from the syringes were connected to the device inlet ports through the microfluidic interface. A syringe pump was used to provide the desired constant volumetric flow rates during the separation process.

The assembled microseparator was placed under a fluorescent microscope and the trajectory of blood cells was monitored and recorded using the microscope camera and video capture tools (Fig. 5). The separation efficiency of the magnetophoretic separation system was characterized by counting the number of the RBCs using a Coulter Cell Counter (Multisizer II, Beckman Coulter, Inc., Fullerton, CA)

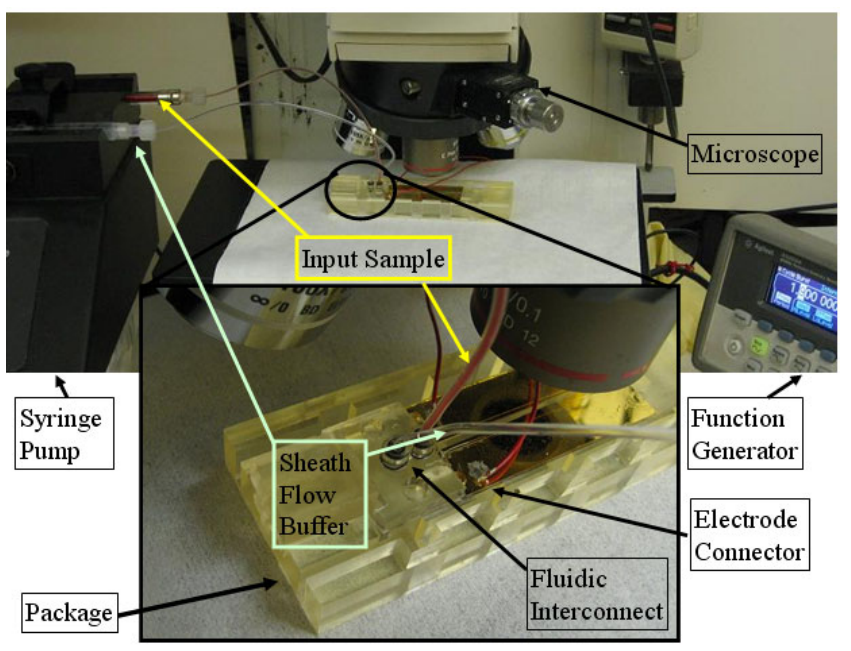

Figure 5: Instrument setup 


\section{RESULTS}

\section{Fabrication Results}

The fabricated angled-electrode type microsystems are shown in Fig. 6. The microfluidic interface with two interconnection ports is shown bonded to the microseparator. Three input channels and comb-type angled electrodes which are centered on the input sample channel and the outlet \#1 channel are shown. Two additional electrode connection holes were drilled to test the electrical connection after silver paste wire bonding. A narrow ditch was etched around the main separation chamber to prevent UV adhesive resin from flooding into the main channel during the bonding process.

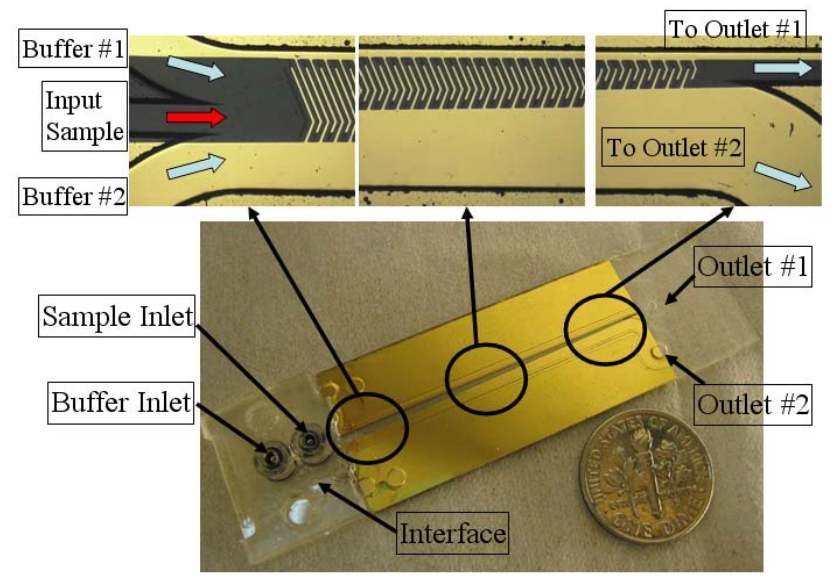

Figure 6: Fabricated hopping mode DEP microsystem

The fabricated divergent and convergent type DEP microseparators are shown in Fig. 7. The punched holes in the PDMS work as the microfluidic interfaces for blood sample introduction.

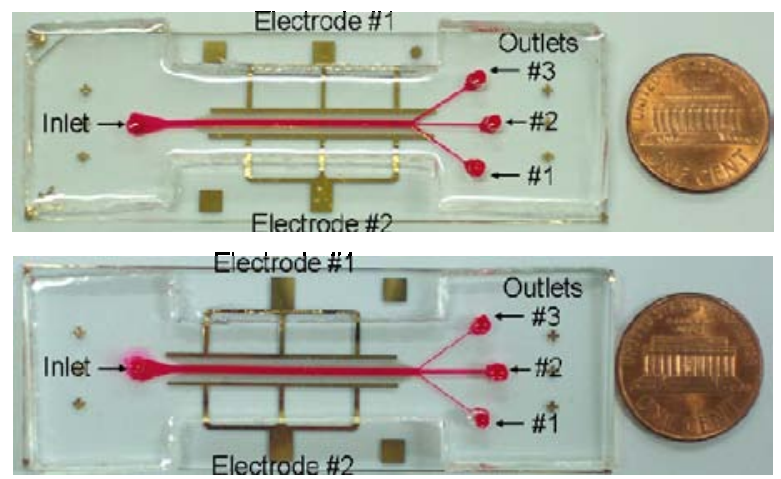

Figure 7: Fabricated convergent and divergent type microseparators

\section{Characterization Results}

The images of a blood cell stream inside the angled-electrode type DEP microsystem with no electric field are shown in Fig. 8 (a). Without an applied DEP force, $99.21 \pm 0.08 \%$ of the cells from the input sample traveled in the laminar flow direction and were collected into outlet \#2. A $2 \mathrm{MHz}, 8 \mathrm{Vp}-\mathrm{p}$ AC signal was supplied in burst mode ( 0.2 second $\mathrm{ON}$ and 1.3 second OFF) to create the hopping movement of the blood cells between adjacent electrodes in the angled-electrode type microsystem. Fig. 8 (b) shows switching of the input blood cell stream from outlet \#2 to outlet \#1. For switching operation, $96.16 \pm 2.48 \%$ cells were deflected from the original flow direction and collected into outlet \#1.

The volumetric flow rate for this demonstration was $20 \mu \mathrm{L} / \mathrm{hr}$ $\left(3.0 \times 10^{3}\right.$ cells/sec). The configuration of the burst mode (periods for release and attraction phase) used for the switching operation was decided for the captured cells to cross one or two electrodes during one period based on the observed cell movement at the fixed volumetric flow rate

A $100 \mathrm{KHz}, 8 \mathrm{~V}$ p-p potential was applied to the electrodes for cell separation. The microscope images of the cell separation in the microsystem demonstrate the separation of WBCs (outlet \#1) and RBCs (outlet \#2). At this condition, most of RBCs did not experience the positive DEP force, and traveled in the laminar flow direction and were collected into outlet \#2, while WBCs showed the positive DEP attraction to the electrode edges and moved toward outlet \#1 (Fig. 9).

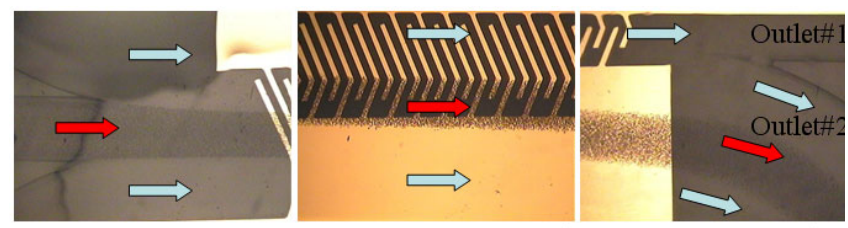

(a) Without Electric Field Buffer Flow $\Longrightarrow$ Blood Flow $\quad \Rightarrow$

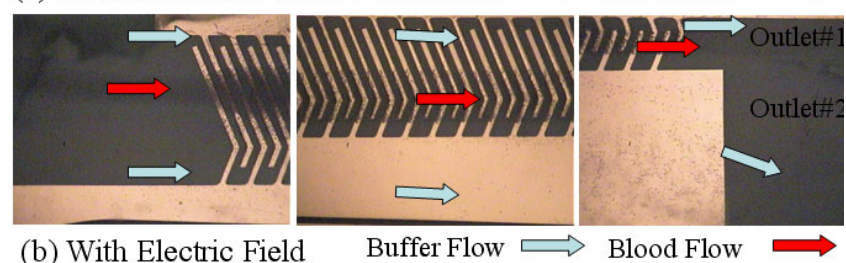

(b) With Electric Field

Buffer Flow $\Longrightarrow$ Blood Flow

Figure 8: Micrograph of blood cells at the entrance, middle and exit of channel

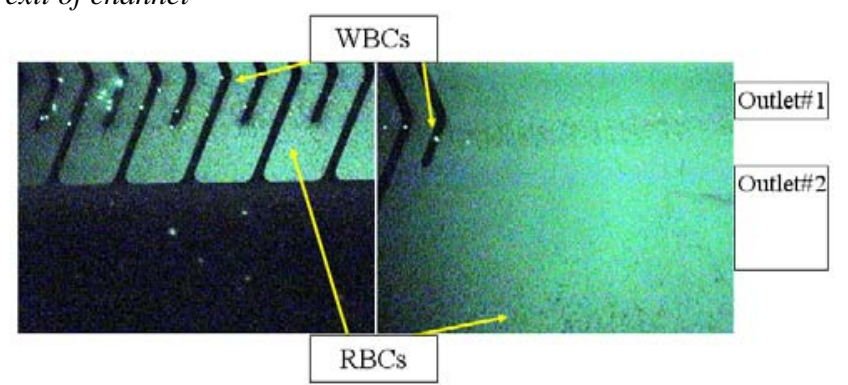

Figure 9: Blood cell separation in the microsystem

$2 \mathrm{MHz}, 3 \mathrm{Vp}-\mathrm{p}$ sinusoidal voltage was applied to separate WBCs from RBCs by the DEP force inside the divergent and convergent type DEP microseparators. Suspending media with a high conductivity of $17 \mathrm{mS} / \mathrm{cm}$ comparable to the conductivity of human whole blood was used for the cell separation. Fig. 10 shows the images of RBCs and fluorescently dyed WBCs passing through the microchannel of the DEP microseparators. In the divergent type microseparator, the RBCs were drawn closer to the edges of the microchannel and flowed into the two outermost outlets (\#1 and \#3), while the WBCs were concentrated at the center of the microchannel and flowed into the central outlet (\#2). In the convergent type microseparator, the RBCs were concentrated toward the center of the microchannel and flowed into the central outlet (\#2), while the WBCs were drawn closer to the edges of the microchannel and flowed into the two outermost outlets (\#1 and \#3). The volumetric flow rate for this cell separation was $50 \mu \mathrm{L} / \mathrm{hr}$ $\left(4.0 \times 10^{4}\right.$ cells/sec $)$. 
(a)

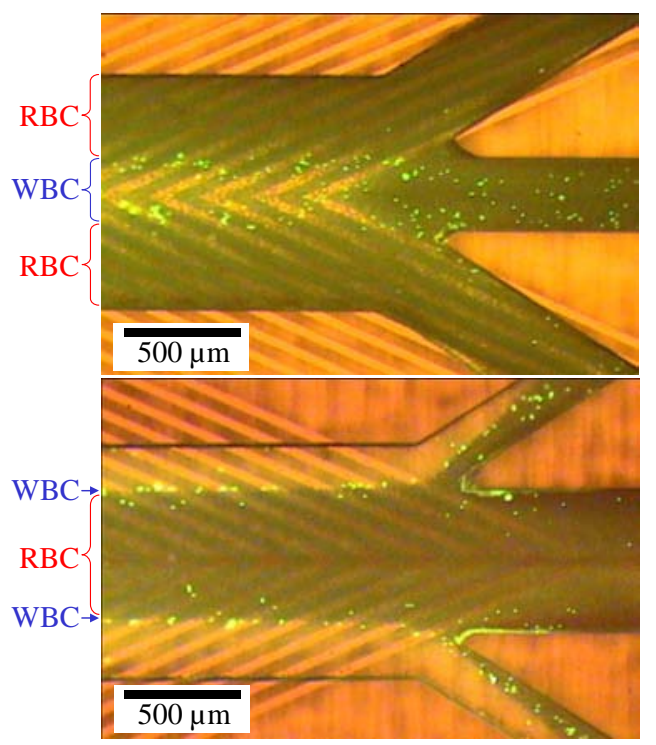

Figure 10: Micrograph of blood cell separation (a) in the divergent and (b) in the convergent type DEP microseparators

With the divergent type DEP microseparator, $87.0 \%$ of RBCs passed through the outermost outlets (\#1 and \#3) while $92.1 \%$ of WBCs were diffused to the central outlet (\#2) (Fig. 11 (a)). With the convergent type DEP microseparator, $93.6 \%$ of RBCs are separated into the central outlet (\#2), while $76.9 \%$ of WBCs diffused to the two side outlets (\#1 and \#3) (Fig. 11 (b)).

(a)

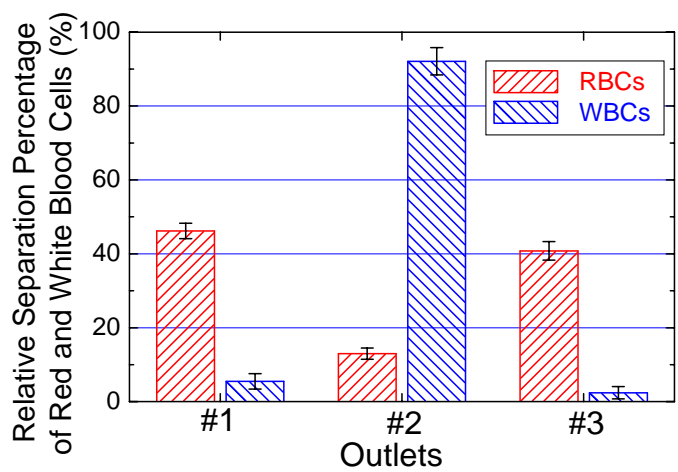

(b)

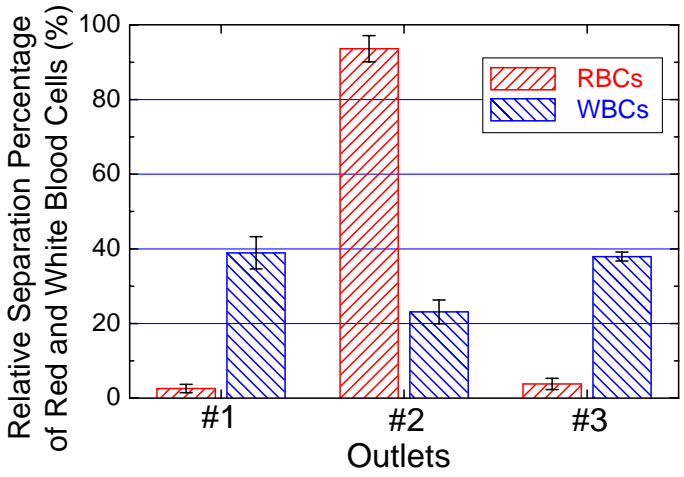

Figure 11: The measured relative separation percentage of $R B C s$ and WBCs at each outlet of the continuous DEP microseparators for (a) divergent type and (b) convergent type design
For the angled-electrode type microsystem, it was observed that some captured cells moved only between the lower edges of the neighboring electrodes and did not change its direction enough toward the electrode center as desired. These cells were sometimes swept toward the undesired outlet. The electrode design that ensures the captured cells to move toward the desired outlet during the attraction phase will help increase the performance efficiency.

No clogging due to cell coagulation inside the chamber was observed during the experiments. However, when the frequency of the applied signal went below $100 \mathrm{~Hz}$ during frequency sweeping, the suspending media started to be electrolyzed and create bubbles inside the chamber, which obstructed the blood stream. At very low volumetric flow rate, unlike most of RBCs, some WBCs adhered to the electrode edge until strong negative force was exercised on the WBCs.

\section{CONCLUSION}

The continuous flow DEP microsystems have successfully demonstrated the ability to switch the flow stream of blood cells between multiple outlet channels and to separate the WBCs from whole blood sample according to their native dielectric properties without the need for tagging or chemical modification of the blood sample. The presented microseparators combined further with miniaturized downstream cell detection and analysis system are expected to enable efficient and novel separation of suspended cells in blood with the potential for portability, reduced fabrication and operation cost, and future development of clinical point-of-care devices for abnormal cell isolation, analysis, and treatment.

\section{REFERENCES}

[1] P. R. C. Gascoyne, J. V. Vykoukal, J. A. Schwartz, T. J. Anderson, D. M. Vykoukal, K. W. Current, C. McConaghy, F. F. Becker, and C. Andrews, "Dielectrophoresis-based programmable fluidic processors," Lab on a Chip, vol. 4, pp. 299-309, 2004.

[2] F. F. Becker, X. B. Wang, Y. Huang, R. Pethig, J. Vykoukal, and P. R. C. Gascoyne, "SEPARATION OF HUMAN BREAST-CANCER CELLS FROM BLOOD BY DIFFERENTIAL DIELECTRIC AFFINITY," Proceedings of the National Academy of Sciences of the United States of America, vol. 92, pp. 860-864, Jan 1995.

[3] S. Fiedler, S. G. Shirley, T. Schnelle, and G. Fuhr, "Dielectrophoretic sorting of particles and cells in a microsystem," Analytical Chemistry, vol. 70, pp. 1909-1915, May 1998.

[4] C. H. Tai, S. K. Hsiung, C. Y. Chen, M. L. Tsai, and G. B. Lee, "Automatic microfluidic platform for cell separation and nucleus collection," Biomedical Microdevices, vol. 9, pp. 533-543, Aug 2007.

[5] I. Doh and Y. H. Cho, "A continuous cell separation chip using hydrodynamic dielectrophoresis (DEP) process," Sensors and Actuators a-Physical, vol. 121, pp. 59-65, May 2005.

[6] Y. L. Li, C. Dalton, H. J. Crabtree, G. Nilsson, and K. Kaler, "Continuous dielectrophoretic cell separation microfluidic device," Lab on a Chip, vol. 7, pp. 239-248, 2007. 